\title{
Two gales do not make a greenhouse
}

Northern Europe, recovering from last week's gales, is ready to believe in a global warming caused by an excess greenhouse effect. But that is not the reason which an international convention must be hurried along.

MANY of the traditionally white clapboard churches of New England are distinguished by their curiously truncated spires, mere stumpy bases which are spires only in the mind's eye. In the locality, the explanation is well-known. For most of the nineteenth century and for much of this, New England was free from Caribbean hurricanes. But then, in 1938, the hurricane track changed, and violent storms that year truncated many pre-existing church spires. Cautious congregations decided to leave things more or less as they were left by the winds, but the more dutiful, who rebuilt, have not been seriously incomoded. Since the 1960s, hurricanes on the East Coast of the United States have followed yet a different track.

That is one comment on the meteorology of northern Europe during the past few weeks. Last Thursday, in particular, winds associated with a deep but narrow depression moving east up the English Channel caused havoc that killed close on fifty people in southwest Britain, The Netherlands and northern West Germany. By the end of the week, the sequence of depressions lining up in the North Atlantic had created a sense of excited caution among Europe's weather forecasters. Similarities with the great wind of October 1987, perhaps technically a hurricane, were too close for comfort.

What has happened to change the usual pattern of winter weather in Northern Europe, when anticyclones can usually be relied upon to anchor themselves over Scandinavia, bringing freezing weather and occasional blizzards? Especially because ski resorts in the Alps have been moaning for months (as they were last year) that there is not enough snow on the lower slopes, it is natural to look for some all-embracing explanation. What, in present circumstances, can it be?

Mrs Margaret Thatcher, the British prime minister, has several faults, impulsiveness the most endearing of them. Last weekend she put into words a thought that has been in knowledgeable people's minds for some time, that the unseasonable weather and now the wind is a sign that the greenhouse effect, once a hypothesis, has become reality. "It is strange to have two hurricanes in this manner", she told an audience at Wigan, in northwest England. "We've also had two mild winters. We don't know whether it's the greenhouse effect yet... but it's a double reminder that we must take every action to keep the outer atmosphere intact."

What Mrs Thatcher said, of course, is strictly proper and entirely correct. It is strange, in the sense of being unfamiliar in northern Europe, that there has been so much high wind, and that two consecutive winters have been exceptionally mild. It was proper of her quickly to imply that these isolated pieces of information do not constitute a proof that the greenhouse effect is already with us, although purists may complain that the word "yet" begs the question. And it is natural that a politician laudably committed to the notion that something drastic will have to be done to fend off the reality of the greenhouse effect should have seized on people's sense of alarm to bend their inclinations to her purpose.

The danger, for prime ministers no less than for others with the environmental bit more professionally between their teeth, is that even indirect and properly qualified assertions that transitory events are evidence for more durable physical phenomena may backfire. Will last weekend's audience put the greenhouse effect at the back of its mind if there should be no near-hurricane in the next winter season, or if dedicated skiers are able to take their exercise without travelling very far?

That hurricanes, or near-hurricanes, may be signs of the reality of an excess greenhouse effect is plausible enough. If it were the case that depressions over the North Atlantic are formed off the eastern coast of North America as a consequence of the contrast of temperature between the land and the sea, and if they are then intensified as a result of the accumulation of thermal energy in the ocean, the events of the past few weeks or even years would be explained (in European eyes) if global warming were already a reality. One might even plead Le Chatellier's principle to argue that, if infrared radiation from the surface of the Earth is impeded by greenhouse gases, the atmospheric system will use other mechanisms for lifting heat in the atmosphere (which depressions accomplish by depositing latent heat at the level of the clouds from whch their precipitation is released).

But the harsh truth is that even this season's string of severe depressions reaching northern Europe is within, if near the extreme of, the recorded pattern of European weather, while there is no evidence that the sea-surface temperatures in the North Atlantic have markedly increased in the past few years. The truth is that the evidence to which Mrs Thatcher drew attention is consistent with, but is not a proof that, there is already an excess greenhouse effect in being. It is equally important that markedly different weather next winter will not be a sign that the threat has gone away.

How, in these circumstances, to create the climate of public opinion that will provide a fair wind for the international conventions to regulate all greenhouse gases for which there is an urgent need? The cumbersome machinery for reaching international agreement is, fortunately, in place. This week's meeting of an international technical panel in Washington (see page 401) is one of several planned in advance of the negotiating session due to be held at Geneva in November.

But it will be a great surprise, and might even be mistaken, if that meeting should arrive at more than a skeleton of a convention. It will be a surprise because the serious political issues that must eventually be tackled - the distribution of carbon dioxide quotas between different countries, for example - have hardly as yet been identified. An agreement would be unwise because - the uncertainties in the opredictions of global warming apart - there remain huge uncertainties about the causes of the accumulation of some of the greenhouse gases, methane, for example, and about the best strategy for abating them.

The importance of tropospheric chlorofluorohydrocabons (CFCs) as greenhouse gases (as distinct from thieves of stratospheric ozone) is well-known, but the economic cost of regulating them is a much less well-known quantity. The 1987 Montreal Protocol to the Vienna Convention on the Safeguarding of the Ozone Layer requires a standstill in production of CFCs, but if there are much less long-lived substitutes in the offing, a complete ban on long-lived chemicals would be more prudent, and cheaper.

That shows that those who draft the next convention will have to create a framework for binding agreement between sovereign states whose details can be changed as knowledge increases and technology advances. That must be a formidable task. Among international conventions, it will be uniquely difficult, which is the chief reason for hurrying.

John Maddox 